Check for updates

1 Policy advisor, The King's Fund, London

2 Policy analyst, The King's Fund, London

Cite this as: $B M J 2021 ; 375: n 2574$ http://dx.doi.org/10.1136/bmj.n2574 Published: 20 October 2021

\title{
Health inequalities and the elective backlog-understanding the problem and how to resolve it
}

\author{
Jonathon Holmes, ${ }^{1}$ Danielle Jefferies ${ }^{2}$
}

Even before covid-19 the NHS was struggling to manage rising demand, as waiting lists continued to grow and people faced long waits for vital treatment. The pandemic has exacerbated the issue and accelerated the rate at which waiting lists were growing.

New analysis from The King's Fund highlights how this trend now risks further widening health inequalities, as people in the most deprived areas are nearly twice as likely to wait over one year for treatment compared to the least deprived. ${ }^{1}$

In July 2021 a total of 5.6 million people were waiting for hospital treatment in England, an increase of 44 per cent compared to April 2020. ${ }^{2}$ With independent estimates suggesting it could more than double to 13 million in the coming months, the secretary of state for health and social care, Sajid Javid has been candid in sharing his expectation that waiting times will get considerably worse before they get better. ${ }^{3}$ In a recent speech, he also spoke about the "social backlog"-the hidden costs of covid and the disproportionate toll it has taken on some of our most disadvantaged communities. ${ }^{4}$

It is difficult to forecast future demand, but looking at where demand has grown most quickly over the course of the pandemic highlights some striking patterns. Firstly, there is a huge variation in rate of growth between different areas. Our analysis shows that some clinical commissioning groups (CCGs) saw an increase of less than 10 per cent, while others saw their waiting list nearly double, with increases of more than 90 per cent. ${ }^{1}$

When we grouped these CCGs by relative deprivation, we found that the most deprived areas saw the greatest rate of increase. ${ }^{5}$ On average the most deprived CCGs saw their waiting lists increase by over half ( 55 per cent) while the least deprived saw increases of around one third ( 36 per cent).

It is important to remember that a growing waiting list is not necessarily a bad thing. It is evidence of referral routes operating effectively and of people coming forward for treatment, which many were reluctant to do during the early waves of the pandemic.

However, looking exclusively at those who had been on the waiting list for over a year, we find an even starker trend. The data shows that 293102 people had been waiting over a year for treatment in July 2021, a 26-fold increase compared to April 2020. ${ }^{1}$

When we looked at this by deprivation we found that people in the most deprived communities are 1.8 times more likely to wait over one year for treatment compared to the least deprived areas.
At a national level, the NHS is clearly aware of the issue and this year's operational planning guidance stresses the importance of tackling the backlog in an inclusive way. 6

While our analysis highlights the scale of the issue, it also points towards possible solutions. For example, using data like this can help identify areas and populations where issues around access are most pronounced. In doing this, health and care systems can prioritise services and communities in a way that is designed to reduce, or at a minimum not exacerbate, health inequalities.

Separately, the government's recent announcement of multi-year resource funding for health and social care, made an additional $€ 8$ billion available to tackle the backlog over the next three years. ${ }^{7}$ In our view it is vital that this money is allocated fairly and that local health and care systems have autonomy to use it flexibly, in a way that is tailored to the specific need of their populations.

With the prospect of a challenging winter and waiting lists growing further, a continued focus on tackling the backlog in an inclusive way will be vital. The pandemic did not create health inequalities but it did exacerbate and highlight them, there is now a choice as the system moves into recovery to work to reduce them as part of this, or to revert to business as usual.

Competing interests: none declared.

Provenance and peer review: commissioned, not peer reviewed.

Mahase E. Most deprived areas in England have fastest growing waiting lists, says think tank. BMJ 2021;374:n2373.

doi: 10.1136/bmj.n2373. pmid: 34580117

2 Consultant-led Referral to Treatment Waiting Times Data 2021-22, NHS England https://www.england.nhs.uk/statistics/statistical-work-areas/rttwaiting-times/rtt-data-2021-22/

3 Sajid Javid's warning of a 13 million NHS waiting list is well within the realms of possibility https://ifs.org.uk/publications/15560

4 The hidden costs of COVID-19: the social backlog https://www.gov.uk/government/speeches/the-hidden-costs-of-covid-19. the-social-backlog

5 English indices of deprivation https://www.gov.uk/government/collections/english-indices-of-deprivation

6 2021/22 priorities and operational planning guidance https://www.england.nhs.uk/publication/2021-22-priorities-and-operational-planning-guidance/

Build Back Better: Our Plan for Health and Social Care

https://www.gov.uk/government/publications/build-back-better-our-planfor-health-and-social-care 